M. Boratyński

Nagoya Math. J.

Vol. 77 (1980), 41-45

\title{
ON A CONJECTURE OF M. P. MURTHY
}

\section{BORATYŃSKI}

In [M] Murthy asked if every subvariety $V$ of $k^{n}$ ( $k$-any field) with a trivial conormal bundle is a (scheme theoretic) complete intersection.

In [B] we were able to prove that all such subvarieties of $k^{n}$ are at least set theoretic complete intersections. Mohan Kumar has shown in [MK] that if one assumes moreover that $n \geq 2 \operatorname{dim} V+2$ then $V$ is a complete intersection. The aim of this paper is to prove the following result which extends the Mohan Kumar Theorem in the nonsingular (and connected) case and moreover sheds some light on his bound for $n$.

Theorem. Suppose $R$ is a noetherian ring with the property that all the (finitely generated) projective $R$-modules are free. Let $P$ be a prime ideal of $A=R[X]$ with $P / P^{2}$ free over $A / P$ of rank $t$ and with $\operatorname{proj} \operatorname{dim} P<\infty$. Then $P$ is a complete intersection ideal if the following three conditions are satisfied:

$1^{\circ} P$ contains monic polynomials

$2^{\circ} \quad R / P \cap R$ is normal

$3^{\circ}$ All the stably free projectives of rank $t-1$ over $A / P$ are free.

Remark. The conditions $1^{\circ}$ and $2^{\circ}$ can be phrased in the following way: The projection $\operatorname{Spec} A \rightarrow \operatorname{Spec} R$ is finite on $\operatorname{Spec} A / P$ and maps Spec $A / P$ onto a normal (closed) subscheme of Spec $R$.

CoRollary 1. Let $V$ be an irreducible subvariety of $k^{n}$ with a trivial conormal bundle. If there exists a finite nonsingular projection of $V$ into $k^{n-1}$ and all the stably free bundles of rank codim $V-1$ over $V$ are trivial then $V$ is a complete intersection.

Corollary 2 (Mohan Kumar). Let $V$ be a nonsingular (connected) subvariety of $k^{n}$ where $K$ is infinite with a trivial conormal bundle. If $n \geq 2 \operatorname{dim} V+2$ then $V$ is a complete intersection.

Received October 31, 1978. 
Proof. It is a folklore that in case $n \geq 2 \operatorname{dim} V+2$ there exists a finite projection $V \rightarrow k^{n-1}$ which imbeds $V$ isomorphically into $k^{n-1}$. So after a change of coordinates we can assume that $A=k\left[X_{1}, X_{2}, \cdots, X_{n}\right]$ $=R[T]$ where $R$ is a polynomial ring over $k$ of $n-1$ indeterminates and $P=I(V)$ satisfies the conditions $1^{\circ}$ and $2^{\circ}$. We have codim $V-1$ $\geq \operatorname{dim} V+1$. By Bass Unimodular Theorem all the stably free bundles of rank codim $V-1$ over $V$ are trivial. Hence the condition $3^{\circ}$ of our Theorem is also satisfied.

CoRollaRy 3. Let $V$ be an irreducible subvariety of $k^{n}$ where $k$ is algebraically closed with a trivial conormal bundle. Suppose that there exists a finite normal projection of $V$ into $k^{n-1}$. If $n \geq 2 \operatorname{dim} V+1$ then $V$ is a complete intersection.

Proof. It suffices to observe that all the stably free bundles of rank codim $V-1$ over $V$ are trivial by [S].

The proof of our Theorem will be preceded by three propositions.

Proposition 1. Let $A=R[X]$ where $R$ is a normal domain and $P$ a prime ideal of $A$ containing monic polynomials. If $P \cap R=(0)$ then $P$ is generated by a monic polynomial.

Proof. Let $K$ be the quotient field of $R$. Then $P K[X]$ is generated by a monic polynomial $f(X)$ in $K[X]$. Since $P$ contains a monic polynomial (with coefficients in $R$ ), the roots of $f$ are integral over $R$. Therefore the coefficients of $f$ are in $R$. Let $g(X) \in P$. Since $f$ divides $g$ in $K[X]$ and since $f$ is monic, the division algorithm shows that $f$ divides $g$ in $R[X]$.

Proposition 2. Let $p$ be an ideal contained in the Jacobson radical of the ring $R$ and let $f$ be any monic polynomial of $R[X]$. Then $p[X]+(f) /(f)$ is contained in the Jacobson radical of $R[X] /(f)$.

Proof. The maximal ideals of $R[X] /(f)$ correspond to the maximal ideals of $R[X]$ which contain $f$. But any such ideal contracts in $R$ to a maximal ideal. So it must contain $p[X]$ since $p$ is contained in the Jacobson radical of $R$. So $p[X]+(f) /(f)$ is contained in the Jacobson radical of $R[X] /(f)$, which was to be proved.

Proposition 3. Let $I$ be an ideal of a ring $A$. Let $f_{1}, f_{2}, \cdots, f_{t}, s, s^{\prime}$ 
be elements of $A$ satisfying the following conditions:

$1^{\circ} f_{1}, f_{2}, \cdots, f_{t}, s^{\prime} \in I$

$2^{\circ} \quad s I \subset\left(f_{1}, f_{2}, \cdots, f_{t}\right)$

$3^{\circ}\left(s, s^{\prime}\right)=A$.

Then $I$ is a homomorphic image of a projective A-module of rank $t$ if the unimodular row $\left[f_{1}, f_{2}, \cdots, f_{t}\right]$ over $A_{s s}$, defines a free module.

Proof. See [B].

Proof of the Theorem. It follows from Proposition 1 that $P=(p[X], f)$ where $f$ is monic in $R[X]$ and $p=P \cap R$. Let $M$ be any maximal ideal of $A$ containing $P$. Suppose $f \in M P$. Then by Nakayama Lemma we have $p[X]_{M}=P_{M}$ which is absurd since $h t p[X]=h t p<h t P(P$ is a nonextended prime ideal). Thus the image of $f$ in $P / P^{2}$ is a unimodular element. By our assumption $P / P^{2}+(f)$ is free over $A / P$. So $v\left(P / P^{2}+(f)\right)=t-1$ where for any module $M v(M)$ denotes its minimal number of generators.

We are now going to apply the techniques which were used independently in [MK] and [B1]. Put $S=1+p$. Then $p_{S}$ is contained in the Jacobson radical of $R_{S}$. Put $\bar{P}_{S}=P_{S} /(f) \subset R_{S}[X] /(f)$. Then $v\left(\bar{P}_{S} / \bar{P}_{S}^{2}\right)$ $=v\left(P_{S} / P_{S}^{2}+(f)\right)=t-1$ since $P_{S} / P_{S}^{2}+(f)$ is a localization of $P / P^{2}+(f)$ which is a free module over $A / P$. By Proposition 2 and Nakayama Lemma $v\left(\bar{P}_{S}\right)=t-1$ since $P_{S}=\left(p_{S}[X], f\right)$. We can assume that $t-1$ elements which generate $\bar{P}_{S}$ are in $\bar{P}=P /(f)$. It follows that $P_{S}=\left(f, f_{2}, \cdots, f_{t}\right)$ where $f, f_{2}, \cdots, f_{t} \in P$. So there exists $s \in S$ such that $s P \subset\left(f, f_{2}, \cdots, f_{t}\right)$. In particular $s(s-1) \in\left(f, f_{2}, \cdots, f_{t}\right)$. So $\left[f, f_{2}, \cdots, f_{t}\right]$ is a unimodular row over $R_{s(s-1)}[X]$ which defines by [Q] the free module since $f$ is monic. It follows from Proposition $3\left(s-1\right.$ plays the role of $\left.s^{\prime}\right)$ that $P$ is a homomorphic image of a projective $A$-module $M$ (say) of rank $t$.

The proof of Proposition 2 in [B] shows that $M$ is free over $R_{s}[X]$ and $R_{s-1}[X]$. So by [Q] $M$ is extended. From our assumption we get that $M$ is free. Thus $v(P) \leq t$ which implies $v(P)=t$ since $r k P / P^{2}=t$. Moreover grade $P=t$ since it is locally so. It follows from [K, Theorem 125] that $P$ is generated by a regular sequence which was to be proved.

Remarks. $1^{\circ}$ Actually what we have done above proves the following

TheOREM. Suppose $R$ is a noetherian ring with all the projectives free. Let $I$ be an ideal of $A=R[X]$ with $I / I^{2}$ free over $A / I$ of rank $t$. Then $I$ 
is a complete intersection if the ideal $I /(I \cap R)[X]$ of $(R / I \cap R)[X]$ is generated by a monic polynomial and all the stably free projectives of rank $t-1$ over $A / I$ are free.

It follows that the connectedness assumption in Corollary 2 is inessential since the Proposition 1 holds in case $R$ is a direct sum of normal domains.

$2^{\circ}$ It follows from Proposition 1 that $P=(p[X], f)$ where $f$ is monic. In view of $[\mathrm{B}$, Lemma 8$]$ the Theorem would be proved if we could show that $v\left(p[X] / p[X]^{2}\right) \leq t-1$. If the regularity assumption on $R$ is added to the assumptions of our Theorem then $p[X] / p[X]^{2}$ is projective of rank $t-1$ over $(R / p)[X]$. It is easy to check that $p[X] / p[X]^{2} \otimes_{(R / p)[X]} A / P \simeq P / p^{2}+(f)$ which is free over $A / P$. So it seems that the only managable information about $p[X] / p[X]^{2}$ we have is that $p[X] / p[X]^{2}$ is an extended projective module over $(R / p)[X]$ whose reduction modulo a monic polynomial is free. Unfortunately one can not conclude from this the freeness of $p[X] / p[X]^{2}$ as the following example shows.

Put $R=R[X, Y, Z] /\left(X^{2}+Y^{2}+Z^{2}-1\right)$. Then

$$
R[T] /\left(T^{2}+1\right)=C[X, Y, Z] /\left(X^{2}+Y^{2}+Z^{2}-1\right) .
$$

Let $M$ denote the projective $R$-module which corresponds to the tangent bundle to $S^{2}$. Then $M[T] \bigotimes_{R[T]} R[T] /\left(T^{2}+1\right)$ is free since all the projectives over $C[X, Y, Z] /\left(X^{2}+Y^{2}+Z^{2}-1\right)$ are free by $[M 1]$.

So it seems that the idea which would considerably shorten our proof does not work in general.

But there is one instance where it works. It is so in case the projection $\operatorname{Spec} A \rightarrow \operatorname{Spec} R$ induces an isomorphism of $\operatorname{Spec} A / P$ with $\operatorname{Spec} R / p$.

Thus the above argument gives the elementary proof of Corollary 2.

The author would like to thank the referee for his proof of Proposition 1 which is stronger than in the original version of this paper.

\section{REFERENCES}

[B] M. Boratyński, A note on the set-theoretic complete intersection ideals, J. of Alg. 54 (1978), 1-5.

[B1] M. Boratyński, A proof of the Eisenbud-Evans conjecture for polynomial rings over a field (unpublished).

[K] I. Kaplansky, Commutative rings, The University of Chicago Press 1974.

[M] M. P. Murthy, Complete intersections, Conference on Commutative Algebra 1975, Queen's University 196-211. 
[M1] M. P. Murthy, Vector bundles over affine surfaces birationally equivalent to ruled surfaces, Ann. of Math. 89 (1969), 242-253.

[MK] N. Mohan Kumar, On two conjectures about polynomial rings, Inv. Math. 46 (1978), 225-236.

[Q] D. Quillen, Projective modules over polynomial rings, Inv. Math. 36 (1976), 167171.

[S] A. A. Suslin, On the stably free modules, Math Sbornik 102 (1977), 537-550 (in Russian).

Institute of Mathematics

Śniadeckich 8, Warsaw

Poland 